\title{
ON THE STABILIZATION OF THE ENERGY OF A HARMONIC OSCILLATOR DISTURBED BY RANDOM PROCESSES OF THE "WHITE AND SHOT NOISES" TYPES
}

\author{
GRIGORI L. KULINICH \\ and SVITLANA V. KUSHNIRENKO \\ Kyiv University \\ Mechanics and Mathematics Faculty \\ 64 Volodymyrska St. \\ Kyiv 252033, Ukraine
}

(Received October, 1998; Revised May, 1999)

In this paper the behavior of the instantaneous energy of a harmonic oscillator is investigated in the case when at a certain angle to the vector of the phase velocity of the oscillator, random disturbances of the "white and shot noises" types are acting.

Key words: Harmonic Oscillator, Instantaneous Energy of the Oscillator, Differential Equation of the Second Order, Stochastic Differential Equation without Aftereffect, Stabilization, Control.

AMS subject classifications: $60 \mathrm{H} 10$.

\section{Introduction}

By harmonic oscillator without friction we mean an oscillating system for which motion is described by the following linear differential equation of the second order

$$
\ddot{u}(t)+k^{2} u(t)=0, \quad u(0)=u_{0}, \quad \dot{u}(0)=\dot{u}_{0},
$$

where $u_{0}$ is the initial position and $\dot{u}_{0}$ is the initial velocity of the oscillator $\left(u_{0}^{2}+\right.$ $\left.\dot{u}_{0}^{2}>0\right) ; k>0$ is a parameter of the oscillator; $u(t)$ is the position and $\dot{u}(t)$ is the velocity of the oscillator at the moment of time $t>0$, and $\varepsilon(t)=\frac{1}{2}\left[k^{2} u^{2}(t)+\dot{u}^{2}(t)\right]$ is the instantaneous energy of the oscillator.

Equation (1) is equivalent to the system of first order differential equations

$$
\left\{\begin{array}{c}
\dot{x}_{1}(t)=k x_{2}(t), \\
\dot{x}_{2}(t)=-k x_{1}(t),
\end{array}\right.
$$


where $\quad x_{1}(t)=k u(t), x_{2}(t)=\dot{u}(t)$. In addition, $2 \varepsilon(t)=|x(t)|^{2}$, where $\quad x(t)=$ $\left(x_{1}(t), x_{2}(t)\right)$.

In the present paper we investigate the behavior of instantaneous energy $\varepsilon(t)$ in the case when, at a certain angle to $b=\left(k x_{2}(t),-k x_{1}(t)\right)$, where $b$ is the vector of the phase velocity of system (2), fluctuations of the "white noise" type $(\dot{w}(t)$ is a "derivative" of a Wiener process $w(t))$ and fluctuations of the "shot noise" type $(\dot{\nu}([0, t), R)$ is a "derivative" of a Poisson measure $\nu([0, t), R))$ are acting. In this case system (2) is considered as the following system of stochastic differential equations without aftereffect (see [2]):

$$
d x(t)=a(t, x(t)) d t+b(t, x(t)) d w(t)+\int_{R} c(t, x(t), u) \nu(d t, d u)
$$

where $a(t, x)=\left(q_{1}(t, x) x_{1}+q_{2}(t, x) x_{2},-q_{2}(t, x) x_{1}+q_{1}(t, x) x_{2}\right)$,

$$
\begin{aligned}
& x=\left(x_{1}, x_{2}\right) \in R \times R, x_{1}(0)=k u_{0}, x_{2}(0)=\dot{u}_{0}, \\
& b(t, x)=\left(g_{1}(t, x) x_{1}+g_{2}(t, x) x_{2},-g_{2}(t, x) x_{1}+g_{1}(t, x) x_{2}\right), \\
& \left.c(t, x, u)=\left(\gamma_{1}(t, x, u) x_{1}+\gamma_{2}(t, x, u) x_{2}\right)-\gamma_{2}(t, x, u) x_{1}+\gamma_{1}(t, x, u) x_{2}\right),
\end{aligned}
$$

$u \in R$ is a non-random vector function, $w(t)$ is a one-dimensional Wiener process, $\nu([0, t), A))$ is a Poisson measure with parameter $t \Pi(A)$, such that $\Pi(R)<\infty$. The process $w(t)$ and the measure $\nu([0, t), A)$, are defined on the probability space $(\Omega, F, P)$. They are jointly independent and $F_{t}$-measurable for any $t \geq 0$ and $A$, where $F_{t} \subset F$ is a nondecreasing family of $\sigma$-algebras.

Qualitative analysis of the behavior of the harmonic oscillator without friction under the random perturbation along the vector of the phase velocity by stochastic process of the "white noise" type is made in paper [5] and qualitative analysis of the behavior of the harmonic oscillator with friction is made in paper [6]. Book [8] gives a formula for the fundamental matrix for linear equations of type (3) with varying coefficients. For equations with constant coefficients, conditions are given under which $|x(t)| \rightarrow 0$ with probability 1 as $t \rightarrow \infty$ as well as conditions under which $E|x(t)|^{2} \rightarrow 0$ as $t \rightarrow \infty$. The behavior of the instantaneous energy of the harmonic oscillator under the random perturbation only of the second component of the vector of the phase velocity was investigated by many authors (see, for example $[3,4,7,9]$ ).

In the present paper, we investigate the sufficient conditions under which the instantaneous energy does not change: $\varepsilon(t)=\varepsilon(0)$ (Corollary 1 of Theorem 1), the sufficient conditions under which the instantaneous energy $\varepsilon(t)$ changes only step-wise (Theorem 2), as well as the sufficient conditions of stability $\varepsilon(t)$ (Theorems 3-5) are established for equation (3) in terms of functions $q_{i}(t, x), g_{i}(t, x), \gamma_{i}(t, x, u)$. It is shown that it is possible to control the behavior of $\varepsilon(t)$ by the choice of function $q_{1}(t, x)$ (determined disturbance).

We will assume that functions $q_{i}(t, x), g_{i}(t, x), \gamma_{i}(t, x, u)$ are such that coefficients of equation (3) satisfy the conditions:

1. $\exists C>0:|a(t, x)|^{2}+|b(t, x)|^{2}+\int_{R}|c(t, x, u)|^{2} \Pi(d u) \leq C\left[1+|x|^{2}\right]$ 
2. $\forall N>0 \exists C_{N}:|a(t, x)-a(t, y)|^{2}+|b(t, x)-b(t, y)|^{2}+\int_{R} \mid c(t, x, u)-$ $\left.c(t, y, u)\right|^{2} \Pi(d u) \leq C_{N}|x-y|^{2}$ with $|x| \leq N,|y| \leq N$

3. $\Pi\{u:|x+c(t, x, u)|=0\}=0$ for all $t \geq 0,|x| \neq 0$.

It is known (see [2]) that conditions 1, 2 guarantee existence of the unique continuation from the right strong solution $x(t)=\left(x_{1}(t), x_{2}(t)\right)$ of equation (3).

In addition, we will use the following designations:

$$
\begin{gathered}
\tilde{\nu}(t, A)=\nu(t, A)-t \Pi(A) ; \\
I(t, x)=2 q_{1}(t, x)+g_{2}^{2}(t, x) ; \\
I_{1}(t, x)=I(t, x)+g_{1}^{2}(t, x) ; \\
I_{2}(t, x)=I(t, x)-g_{1}^{2}(t, x) ; \\
\psi(t, x, u)=\left(1+\gamma_{1}(t, x, u)\right)^{2}+\gamma_{2}^{2}(t, x, u) .
\end{gathered}
$$

\section{Stabilization of $\varepsilon(t)$}

According to the generalized Ito's formula (see [2]):

$$
\begin{aligned}
d|x(t)|^{2}= & {\left[2(x(t), a(t, x(t)))+|b(t, x(t))|^{2}\right] d t+2(x(t), b(t, x(t))) d w(t) } \\
& +\int_{R}\left[|x(t)+c(t, x(t), u)|^{2}-|x(t)|^{2}\right] \nu(d t, d u)
\end{aligned}
$$

where $(\cdot, \cdot)$ is the inner product.

Thus,

$$
\begin{aligned}
d|x(t)|^{2} & =|x(t)|^{2}\left\{I_{1}(t, x(t)) d t+2 g_{1}(t, x(t)) d w(t)\right. \\
& \left.+\int_{R}[\psi(t, x(t), u)-1] \nu(d t, d u)\right\} .
\end{aligned}
$$

Condition 3 implies that $\psi(t, x, u)>0$ in measure $\Pi(d u)$ for all $t \geq 0, x$. Therefore (see [8]):

$$
\begin{gathered}
|x(t)|^{2}=|x(0)|^{2} \exp \left\{\int_{0}^{t} I_{2}(s, x(s)) d s+2 \int_{0}^{t} g_{1}(s, x(s)) d w(s)\right. \\
\left.\int_{0}^{t} \int_{R} \ln \psi(s, x(s), u) \nu(d s, d u)\right\} .
\end{gathered}
$$

Relations (4) and (5) imply the following statements.

Theorem 1: If for all $t \geq 0$ and $x$ 
(1) $g_{1}(t, x)=0$;

(2) $\Pi\{u: \psi(t, x, u) \neq 1\}=0$,

then with probability 1 for all $t \geq 0$ the following inequality holds true:

$$
|x(0)|^{2} e^{\int_{0}^{t} m(s) d s} \leq|x(t)|^{2} \leq|x(0)|^{2} e^{\int_{0}^{t} M(s) d s}
$$

where

$$
m(t)=\inf _{x} I(t, x), \quad M(t)=\sup _{x} I(t, x) .
$$

Proof: Therefore, in this case relation (5) takes the form

$$
|x(t)|^{2}=|x(0)|^{2} \exp \left\{\int_{0}^{t} I(s, x(s)) d s\right\}
$$

which implies the statement of Theorem 1.

Remark 1: Condition (1) means that system (2) is perturbed by "white noise" only along the vector of the phase velocity. It follows from condition (2) that

$$
2(x, c(t, x, u))=-|c(t, x, u)|^{2}<0
$$

in measure $\Pi(d u)$ for all $t \geq 0,|x| \neq 0$. Thus, condition (2) means that system (2) is perturbed by "shot noise" at an obtuse angle to the radius-vector.

Corollary 1: Under conditions (1) and (2) of Theorem 1 it is possible to control the behavior of $\varepsilon(t)$ of a perturbed system by the choice of function $q_{1}(t, x)$ (determined disturbance). For example:

(1) if $2 q_{1}(t, x)=-g_{2}^{2}(t, x)$, then $\varepsilon(t)=\varepsilon(0)$ with probability 1 for all $t \geq 0$;

(2) if $\left|\int_{0}^{t} M(s) d s\right| \leq C$, then $\varepsilon(t) \leq \varepsilon(0) e^{C}$ with probability 1 for all $t \geq 0$;

(3) if $2 q_{1}(t, x)=-g_{2}^{2}(t, x)+c_{0}$, then $\varepsilon(t)=\varepsilon(0) e^{c_{0} t}$; etc.

Theorem 2: If $\gamma_{i}(t, x, u)=\gamma_{i}(t, u), i=1,2$ and $g_{1}(t, x)=0,2 q_{1}(t, x)+g_{2}^{2}(t, x)=0$ for all $t \geq 0$ and $x$, then

$$
|x(t)|^{2}=\left\{\begin{array}{cc}
|x(0)|^{2}, & \text { if } t<\tau_{1}, \\
|x(0)|_{\tau_{k}<t}^{2} \prod_{k} \psi\left(\tau_{k}, u_{k}\right), & \text { if } \tau_{k} \leq t<\tau_{k+1},
\end{array}\right.
$$

where $0<\tau_{1}<\tau_{2}<\ldots$ are shock-points of a Poisson process $\nu([0, t), R)$ and $\nu\left(\left\{\tau_{k}\right\}\right.$, $\left.\left\{u_{k}\right\}\right)=1, k=1,2 \ldots$

Proof: Therefore, under the conditions of Theorem 2, relation (5) takes the following form:

$$
|x(t)|^{2}=|x(0)|^{2} \exp \left\{\int_{0}^{t} \int_{R} \ln \psi(s, u) \nu(d s, d u)\right\}
$$

which implies equality (6) (see [2]).

Corollary 2: Under the conditions of Theorem 2, $\varepsilon(t)$ changes only step-wise: moreover, shocks take place only in the moments of impulse disturbance, that is, in the moments of jumps of a Poisson process $\nu([0, t), R)$. In particular, if 
$\left(1+\gamma_{1}\right)^{2}+\gamma_{2}^{2}$ is a constant magnitude, then

$$
|x(t)|^{2}=|x(0)|^{2}\left[\left(1+\gamma_{1}\right)^{2}+\gamma_{2}^{2}\right]^{\nu((0, t], R)} .
$$

Remark 2: If $\Pi\left\{u:\left(1+\gamma_{1}(t, u)\right)^{2}+\gamma_{2}^{2}(t, u) \neq 0\right\}=0$ for all $t \geq 0$, then

$$
|x(t)|^{2}=\left\{\begin{array}{cl}
|x(0)|^{2}, & \text { if } t<\tau_{1} \\
0, & \text { if } \tau_{1} \leq t
\end{array}\right.
$$

This means that under the first impulse disturbance, the considered system moves into the equilibrium state and does not leave it with probability 1 . Thus in this case with small disturbances of coefficients $\gamma_{i}(t, u)$, it is possible to achieve equality (7) and then obtain (8) by passing to the limit.

Theorem 3: If for all $t \geq 0$

then

$$
\sup _{x}\left[I_{1}(t, x)+\int_{R}[\psi(t, x, u)-1] \Pi(d u)\right] \leq 0
$$

$$
P\left\{\sup _{t \geq 0}|x(t)|^{2}<\varepsilon_{1}\right\} \geq 1-\varepsilon_{2}
$$

for any $\varepsilon_{1}>0, \varepsilon_{2}>0$ as soon as $|x(0)|<\delta ; \delta>0$.

Proof: Formula (4) implies the following equality:

$$
|x(t)|^{2}=|x(0)|^{2}+\int_{0}^{t}|x(s)|^{2}\left\{I_{1}(s, x(s))+\int_{R}[\psi(s, x(s), u)-1] \Pi(d u)\right\} d s+\eta(t),
$$

where

$$
\eta(t)=2 \int_{0}^{t} g_{1}(s, x(s)) d w(s)+\int_{0}^{t}[\psi(s, x(s), u)-1] \widetilde{\nu}(d s, d u) .
$$

Therefore, with probability 1 for all $t \geq 0$

$$
|x(t)|^{2} \leq|x(0)|^{2}+\eta(t)
$$

Since $\eta(t)$ is a square integrable martingale, then from the inequality (9) we have (see $[1])$ :

$$
P\left\{\sup _{t \geq 0} \eta(t)>\varepsilon_{1}\right\} \leq \frac{|x(0)|^{2}}{\varepsilon_{1}} .
$$

The statement of Theorem 3 follows from (9) and (10).

Theorem 4: If

(1)

$$
\varlimsup_{t \rightarrow \infty} \frac{1}{t^{\alpha}} \int_{0}^{t} \sup _{x}\left[I_{2}(s, x)+\int_{R} \ln \psi(s, x, u) \Pi(d u)\right] d s<0
$$


(2)

for some $\alpha>\frac{1}{2}$; and

$$
\text { then } \begin{gathered}
\int_{0}^{t} \sup _{x}\left[4 g_{1}^{2}(s, x)+\int_{R} \ln ^{2} \psi(s, x, u) \Pi(d u)\right] \leq C_{0} t \\
P\left\{\lim _{t \rightarrow \infty}|x(t)|^{2}=0\right\}=1 .
\end{gathered}
$$

Proof: Hence, we can rewrite equality (5) as

where

$$
\begin{gathered}
|x(t)|^{2}=|x(0)|^{2} \exp \left\{t ^ { \alpha } \left[\frac { 1 } { t ^ { \alpha } } \int _ { 0 } ^ { t } \left(I_{2}(s, x(s))+\right.\right.\right. \\
\left.\left.\left.+\int_{R} \ln \psi(s, x(s), u) \Pi(d u)\right) d s+\frac{1}{t^{\alpha}} \zeta(t)\right]\right\}
\end{gathered}
$$

$$
\zeta(t)=2 \int_{0}^{t} g_{1}(s, x(s)) d w(s)+\int_{0}^{t} \int_{R} \ln \psi(s, x(s), u) \widetilde{\nu}(d s, d u) .
$$

According to Condition (1) of Theorem 4, we will find $\delta>0$ and $\theta_{\delta}>0$ such that with probability 1

$$
\frac{1}{t^{\alpha}} \int_{0}^{t}\left[I_{2}(s, x(s))+\int_{R} \ln \psi(s, x(s), u) \Pi(d u)\right] d s \leq-\delta,
$$

as $t>T_{\delta}$. Furthermore, since $\zeta(t)$ is a square integrable martingale with characteristics which satisfy the following inequality:

$$
\langle\zeta(t)\rangle=\int_{0}^{t}\left[4 g_{1}^{2}(s, x(s))+\int_{R} \ln ^{2} \psi(s, x(s), u) \Pi(d u)\right] d s \leq C_{0} t .
$$

Reasoning similarly to [4, Lemma 7.1$]$, it can be proved that

$$
P\left\{\lim _{t \rightarrow \infty} t^{-\alpha} \zeta(t)=0\right\}=1
$$

Therefore, taking into account (11), we obtain the statement of Theorem 4 .

Theorem 5: If for all $t \geq 0$ and $x$ :

and

$$
I_{1}(t, x)+\int_{R}[\psi(t, x, u)-1] \Pi(d u)=Q(t)
$$

then

$$
\varlimsup_{t \rightarrow \infty} \int_{0}^{t} Q(s) d s=-\infty
$$




$$
\lim _{t \rightarrow \infty} E|x(t)|^{2}=0
$$

Proof: Hence, from relation (4) we have

$$
E|x(t)|^{2}=|x(0)|^{2}+\int_{0}^{t} Q(s) E|x(s)|^{2} d s .
$$

Therefore,

$$
E|x(t)|^{2}=|x(0)|{ }^{2} e^{\int_{0}^{t} Q(s) d s}
$$

This equality implies the statement of Theorem 5 .

Remark 3: If the system is perturbed by "centralized shot noise" $(\dot{\widetilde{\nu}}([0, t), A)$ is a "derivative" of a Poisson nature) instead of "shot noise" and other perturbations are fixed then only the orientation of $a(t, x)$ changes in equation (3), that is,

$$
a(t, x)=\left(\widehat{q}_{1}(t, x) x_{1}+\widehat{q}_{2}(t, x) x_{2},-\widehat{q}_{2}(t, x) x_{1}+\widehat{q}_{1}(t, x) x_{2}\right),
$$

where

$$
\widehat{q}_{i}(t, x)=q_{i}(t, x)-\int_{R} \gamma_{i}(t, x, u) \Pi(d u) .
$$

\section{References}

[1] Gikhman, I.I., Differential equations with random functions, Winter School on the Theory of Probability and Math Statistics (1964), 41-85.

[2] Gikhman, I.I. and Skorokhod, A.V., Stochastic Differential Equations and Their Applications, Naukova Dumka, Kiev 1982.

[3] Gikhman, I.I. and Skorokhod, A.V., The Theory of Stochastic Processes, III, Nauka, Moscow 1975. English Trans. Springer-Verlag, Berlin 1978.

[4] Khasminskii, R.Z., Stability of Systems of Differential Equations under Random Perturbations of the Parameters, Izdat. Nauka, Moscow 1969. English Trans. Kluwer Academic Pubs., Norwell, MA 1980.

[5] Kulinich, G.L., Qualitative analysis of the influence of random perturbations on the phase velocity of the harmonic oscillator, Random Operators and Stoch. Eqns. 3:2 (1995), 141-152.

[6] Kulinich, G.L., Qualitative analysis of the influence of random perturbations of the "white noise" type along the vector of the phase speed on the harmonic oscillator with friction, Ukrain. Math. J. 49:1 (1997), 36-47.

[7] Kulinich, G.L., On the limiting behavior of a harmonic oscillator with random external disturbance, JAMSA 8:3 (1995), 265-274.

[8] Skorokhod, A.V., Asymptotic Methods in the Theory of Stochastic Differential Equations, Naukova Dumka, Kiev 1987. English Trans. Translations of Math. Monographs 78, AMS, Providence, RI 1989.

[9] Stratonovich, R.L., Selected Questions in the Theory of Fluctuations in Radio Engineering, Soviet Radio, Moscow 1961. 


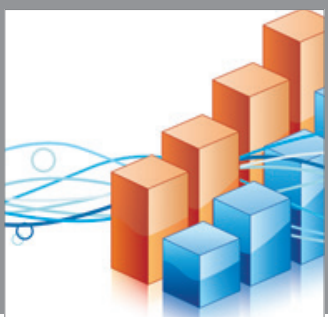

Advances in

Operations Research

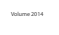

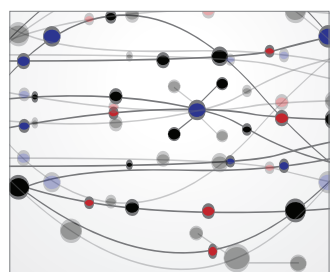

\section{The Scientific} World Journal
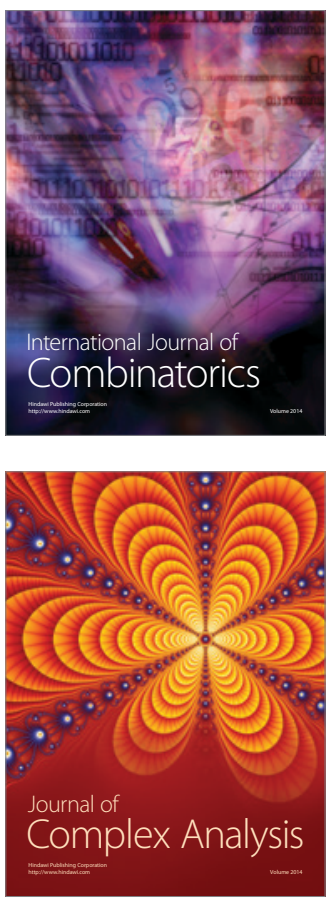

International Journal of

Mathematics and

Mathematical

Sciences
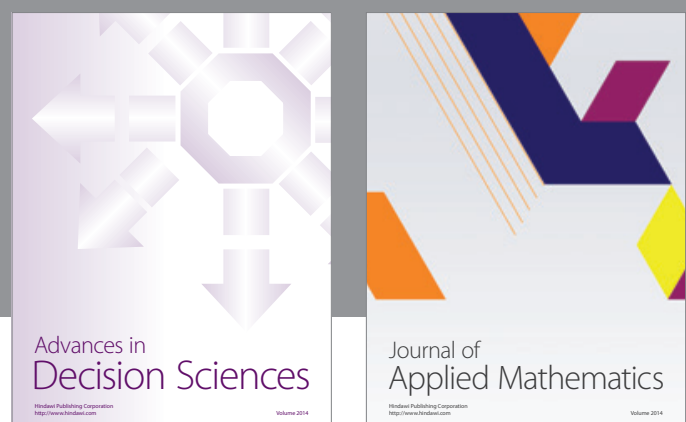

Journal of

Applied Mathematics
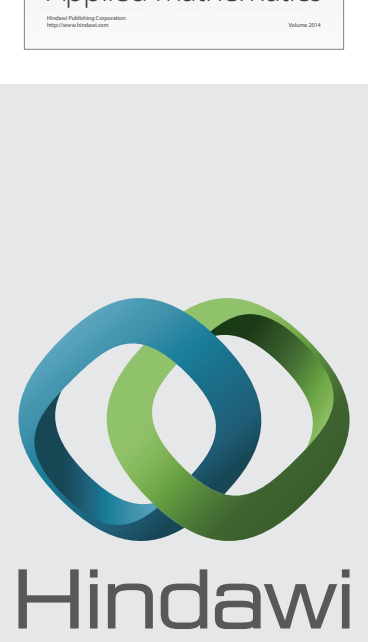

Submit your manuscripts at http://www.hindawi.com
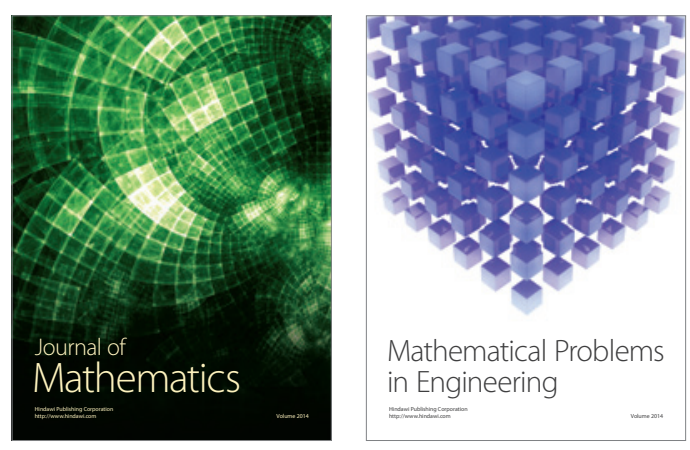

Mathematical Problems in Engineering
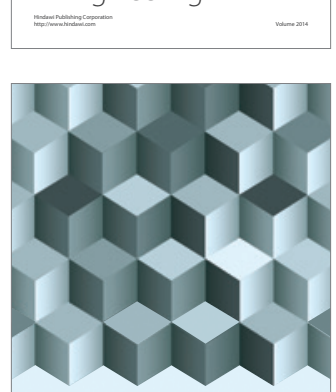

Journal of

Function Spaces
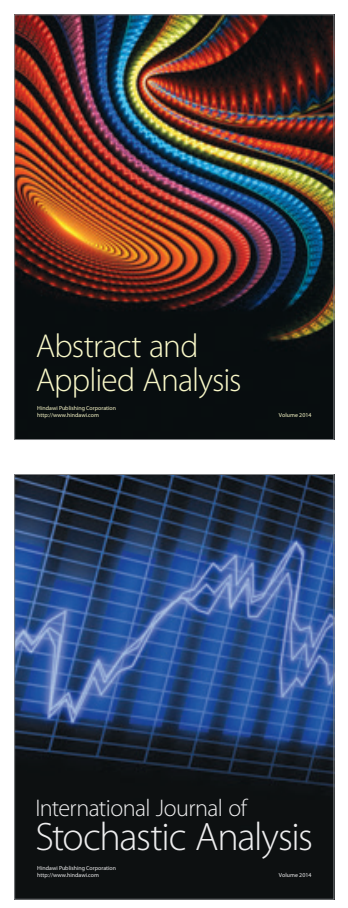

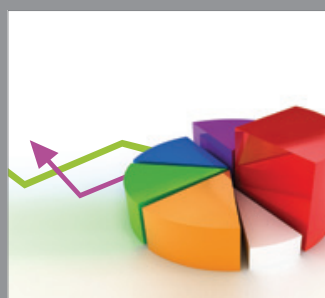

ournal of

Probability and Statistics

Promensencen
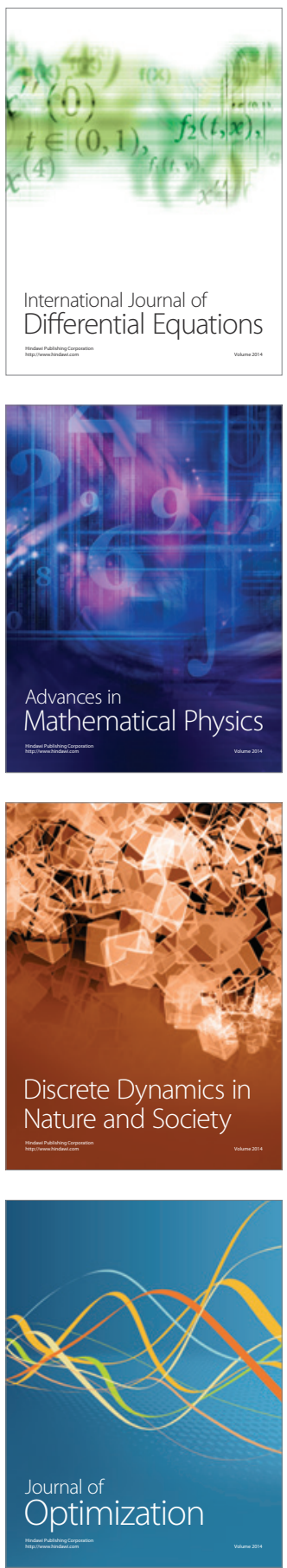